Mitteilungen der Deutschen Gesellschaft fur Phlebologie

\title{
Thrombose unter Corona, wir müssen aktiv werden!!
}

Über Corona nachdenken, das ist kein „alter Hut“, sondern so aktuell wie nie zuvor. Alle haben auf die Impfung gehofft, ihre Umsetzung und dadurch ihre Wirkung sind aber noch sehr schleppend... Die Krankheit an sich und der Lockdown haben nicht nur menschliche Folgen in viel Leid, Einsamkeit und Angst, sondern auch gesellschaftliche, wirtschaftliche, kulturelle...

\section{Was können wir, wir Phlebologen, heute tun?}

Nach 12 Monaten Forschung, die bereits in Wuhan (China) sehr intensiv begonnen hat, ist noch keine spezifische, wirksame Behandlungsoption gefunden. Wir wissen nur, dass das Virus am Ende unser Immunsystem triggert zu Reaktionen, die uns schaden und Menschen dann in einem scheinbar unaufhaltsamen Prozess umbringen.

Die Autopsien aus Italien zeigten bereits im März/April 2020, dass die Thrombosen dabei eine große Rolle spielen. Und zwar nicht nur die gehäuft auftretenden Thrombosen in den Beinvenen mit Lungenembolien, sondern auch arterielle Thrombosen, besonders in den Lungenarterien, aber auch in allen anderen Organen sind möglich. Bereits in Wuhan hatte man eine Verringerung der Sterblichkeit schwerstkranker Patienten auf Intensivstationen unter Heparin von $60 \%$ ohne auf $42 \%$ mit Heparin [1].

Die Gesellschaf für Thrombose und Hämostaseforschung (GTH) empfahl hier in Deutschland bereits im April, bei CoronaInfizierten auch im ambulanten Bereich großzügig Heparin einzusetzen: „Bei allen Patienten mit gesicherter SARS-CoV-2 Infektion sollte die Indikation zur medikamentösen VTE-Prophylaxe mit NMH unabhängig von der
Notwendigkeit einer Hospitalisierung fortlaufend geprüft und großzügig gestellt werden“ (VTE = venöse Thromboembolie; $\mathrm{NMH}=$ niedermolekulares Heparin) [2] (wir berichteten auch hier). Als einzige AWMF-Leitlinie bezieht sich die AWMF-Leitlinie Neues Coronavirus - Informationen für die hausärztliche Praxis, Registernummer 053-054 auf ambulante Patienten. Als einziges Medikament wird hier Heparin empfohlen bei Patienten mit Risikofaktoren wie Adipositas, Alter und vielen Krankheiten [3].

Allerdings sollte dieser Zusammenhang in Studien belegt werden, so forderte es auch die UIA (Union international de Angiologie) im September: „The use of low molecular weight heparin in early stage of the disease could prevent vascular complications and reduce the progression to severe stage of the disease" [4]. Studien zur Wirksamkeit von Heparin [5-12] zur Senkung der Todesrate gibt es schon lange, allerdings nur bei $\mathrm{Pa}$ tienten im Krankenhaus, die bereits schwer krank sind. Wenn Heparin NACH der stationären Aufnahme mit bereits vorliegender Luftnot verabreicht wurde, wird die Sterberate gesenkt, ca. um ein Drittel. Heparin gehört deshalb bereits seit Mai/Juni 2020 international zum Standard der Behandlung von Patienten mit Corona in kritischen Phasen.

Im Juni 2020 als Preprint [13] und im November als Artikel [14] wurde bekannt, dass $52 \%$ der Patienten, die mit Corona auf Intensivstation liegen, ein Antiphospholipid-Syndrom vorweisen, sprich Antiphospholipid-Antikörper im Blut haben. Beim Lupus liegt dieser Prozentsatz zwischen 16 und $35 \% ! !$

Fassen wir also zusammen:

- Heparin hilft, die Todesrate nach stationärer Aufnahme zu senken.
- Es liegen arterielle Thrombosen vor, besonders in den Lungenarterien, die zur bekannten Luftnot führen.

- Corona triggert die Ausschüttung von Antiphospholipid-Antikörpern.

- Die Empfehlung der DGA vom April [3] sowie 2 Artikel aus dem Ärzteblatt zu dem Thema $[15,16]$ verpufften.

Das bedeutet,

- dass sehr häufig mögliche Hilfe in Form von Heparin auch bei ambulanten Patienten gegen das Fortschreiten der Krankheit ausbleibt.

- dass ASS 100, gegeben nach Ansteckung, der arteriellen Thrombose vorbeugen könnte, da dies beim Antiphospholipid-Syndrom der Thrombose ausreichend vorbeugt.

Als Heparin käme zulassungskonform nur das Dalteparin (Fragmin ${ }^{\circledR} \mathrm{P}$ forte) infrage, das zugelassen ist unter anderem für internistische Erkrankungen mit eingeschränkter Bettlägerigkeit; alle anderen Zulassungen beziehen sich nur auf perioperative Prophylaxe oder Bettlägerigkeit.

\section{Was brauchen wir?}

Naheliegend wäre, die Forschung zu Heparin und ASS in Phase II der Corona-Krankheit (ambulanter Patient, leichte Symptome) SOFORT umzusetzen, was derzeit in Deutschland an den extrem hohen Hürden des Arzneimittelgesetztes scheitert [17]. An die Regierung oder Personen im Dunstkreis der Entscheidungsträger kommt man ganz schwer heran, die eine Aufhebung der extrem hohen Auflagen durch die CoronaNotstand-Regelungen ermöglichen könnten. Versuche meinerseits verpufften bisher im Nichts. 


\section{Als Phlebologe selbst aktiv werden!!}

Heparin bei bettlägerigen Corona-Patienten? Diese Information ist verpufft. ASS erwägen? Zur Prophylaxe arterieller Thrombosen? Nichts dergleichen passiert, auch wenn es eigentlich komplett naheliegend ist. Und wir, wir Phlebologen, sind es, die das sehen und diese Information transportieren müssten. Als gesellschaftlicher Auftrag!! Informieren Sie alle Ihre bekannten Kanäle, Ärztenetze, Printmedien, Kollegen, Patienten!

\section{Wir als Phlebologen wissen Bescheid und dürfen nicht länger inaktiv bleiben!}

\section{Dr. Erika Mendoza}

\section{Literatur}

[1] Guan W], Ni ZY, Hu Y et al. Clinical Characteristics of Coronavirus Disease 2019 in China. N Engl J Med 2020; 382 (18): 1708-1720. doi:10.1056/ NEJMoa2002032. Epub 2020 Feb 28. PMID: 32109013

[2] https://www.dga-gefaessmedizin.de/ fileadmin/content/PDFs/Stellungnahmen/ DGA-Stellungnahme_zu_COVID-19-Infektion_ und_VTE-Risiko_.pdf
[3] https://www.awmf.org/leitlinien/detail/II/ 053-054.html

[4] Costanzo L, Failla G, Antignani PL et al. The vascular side of COVID-19 disease. Position paper of the International Union of Angiology. Int Angiol 2020; 39 (6): 445-451. doi:10.23736/ S0392-9590.20.04539-3. Epub 2020 Sep 7. PMID: 32892615

[5] Huang C, Wang Y, Li X et al. Clinical features of patients infected with 2019 novel coronavirus in Wuhan, China. Lancet 2020; 395: 497-506

[6] Tang N, Li D, Wang X et al. Abnormal coagulation parameters are associated with poor prognosis in patients with novel coronavirus pneumonia. J Thromb Haemost 2020 [Epub ahead of print]

[7] Klok FA, Kruip MJHA, van der Meer NJM et al. Incidence of thrombotic complications in critically ill ICU patients with COVID-19. Thromb Res 2020 [Epub ahead of print]

[8] Cui S, Chen S, LiX et al. Prevalence of venous thromboembolism in patients with severe novel coronavirus pneumonia. J Thromb Haemost 2020 [Epub ahead of print]

[9] Tang N, Bai H, Chen X et al. Anticoagulant treatment is associated with decreased mortality in severe coronavirus disease 2019 patients with coagulopathy. J Thromb Haemost 2020 [Epub ahead of print]

[10] Thachil J, Tang N, Gando S et al. ISTH interim guidance on recognition and management of coagulopathy in COVID-19. J Thromb Haemost 2020 [Epub ahead of print]
[11] Casini A, Alberio L, Angelillo-Scherrer A et al. Thromboprophylaxis and laboratory monitoring for in-hospital patients with COVID-19 a Swiss consensus statement by the Working Party Hemostasis. Swiss Med Wkly 2020; 150: w20247

[12] Marietta M, Ageno W, Artoni A et al. COVID-19 and haemostasis: a position paper from Italian Society on Thrombosis and Haemostasis (SISET). Blood Transfus 2020 [Epub ahead of print]

[13] Zuo Y, Estes SK, Gandhi AA et al. Prothrombotic antiphospholipid antibodies in COVID-19. medRxiv [Preprint] 2020. doi:10.1101/ 2020.06.15.20131607. PMID: 32587992; PMCID: PMC7310650

[14] Zuo Y, Estes SK, Ali RA et al. Prothrombotic autoantibodies in serum from patients hospitalized with COVID-19. Sci Transl Med 2020; 12 (570): eabd3876. doi:10.1126/scitranslmed. abd3876. Epub 2020 Nov 2. PMID: 33139519; PMCID: PMC7724273

[15] https://www.aerzteblatt.de/archiv/214218/ Thrombotische-KomplikationenGerinnungsneigung-und-COVID-19

[16] https://www.aerzteblatt.de/archiv/216916/ COVID-19-Evidenzbasierte-Therapieoptionen

[17] Mendoza E. Krankheitsprogression in frühen Stadien von COVID-19 und Gerinnungshemmer: Die Möglichkeiten und Schwierigkeiten einer sinnvollen Untersuchung. Phlebologie 2020; 49: 199-203 